\title{
COMO O ORTOPEDISTA BRASILEIRO TRATA ENTORSE LATERAL AGUDA DO TORNOZELO?
}

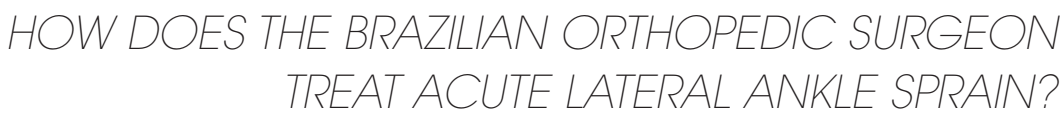

Paulo Santoro Belangero', Marcel Jun Sugawara Tamaoki², Gilberto Yoshinobu Nakama ${ }^{3}$, Marcus Vinicius Shoiti ${ }^{4}$ Rodrigo Vick Fernandes Gomes ${ }^{4}$, João Carlos Belloti ${ }^{5}$

\section{RESUMO}

Objetivo: A entorse lateral aguda do tornozelo (ELAT) é uma afecção frequente cujo tratamento ainda não se encontra totalmente estabelecido. O objetivo do estudo foi verificar a conduta do médico ortopedista brasileiro (incluindo residentes) em relação ao diagnóstico, classificação, tratamento e complicações da entorse lateral aguda do tornozelo (ELAT). Métodos: Um questionário de múltipla escolha foi elaborado com objetivo de abordar os principais aspectos do tratamento da ELAT. O questionário foi veiculado na página eletrônica oficial da Sociedade Brasileira de Ortopedia e Traumatologia, no período de 15 de junho a 1으 de agosto de 2004. Resultados: Foram incluídos para análise um total de 444 questionários. Os resultados demonstraram concordância da maioria dos entrevistados em relação aos seguintes aspectos: 90,8\% utilizam alguma classificação para nortear o tratamento da entorse; $59 \%$ classificam a ELAT com segurança; 63,7\% utilizam imobilização rígida nas lesões ligamentares completas; 60,6\% utilizam medicação anti-inflamatória na ruptura ligamentar parcial; 75,9\% relataram que a dor residual é a complicação mais frequente. Não houve consenso quanto ao método de imobilização da ELAT parcial visto que imobilização e tratamento funcional foram escolhidos com a mesma frequência (47\%). Não houve diferenças significativas entre as respostas dos residentes e a dos ortopedistas $(\mathrm{p}=0,81)$. Conclusões: Os ortopedistas e residentes em ortopedia do Brasil têm dificuldade em classificar a ELAT e não há consenso quanto à melhor opção para a ELAT parcial.

Descritores - Ligamentos Laterais do Tornozelo; Entorses e Distensões; Avaliação em Saúde

\section{ABSTRACT}

Objective: Acute lateral ankle sprain (ALAS) is one of the most common injuries, the treatment of which has yet to be firmly established. The purpose of this study was to determine the Brazilian Orthopaedic Surgeon's behavior in relation to diagnosis, classification, treatment and complications of the Acute Lateral Ankle Sprain. Methods: A multiple choice questionnaire was developed which addressed the main aspects related to the treatmentof acute lateral ankle sprains (ALAS). The questionnaire was made available from June 15 to August 1, 2004, at the Official site of the Brazilian Society of Orthopedics and Traumatology. Results: 444 questionnaires were included in the analysis. The results showed agreement among most of those interviewed in the following regards: $90.8 \%$ use some classification to guide treatment of the sprain; 59\% classify the ankle sprain with certainty; $63.7 \%$ use the immobilization in cases of totally ruptured ligaments; $60.6 \%$ use anti-inflammatory medication in partial ligament ruptures; $75.9 \%$ reported that residual pain was the most frequent complication. There was no consensus regarding treatment of partial ALAS, as immobilization and functional treatment were chosen with the same frequency (47\% each). There was no significant difference between the answers of residents and orthopedists. Conclusions: Orthopedic surgeons and orthopedic residents in Brazil have difficulty classifying ALAS and there is no consensus about the best therapeutic option for partial ALAS.

Keywords - Lateral Ligament, Ankle; Sprains and Strains; Health Evaluation

\footnotetext{
1 - Residente de segundo ano do Departamento de Ortopedia e Traumatologia da Universidade Federal de São Paulo.

2 - Médico Ortopedista do Setor de Ombro e Cotovelo do Departamento de Ortopedia e Traumatologia da Universidade Federal de São Paulo.

3 - Médico Ortopedista do Setor de Joelho do Departamento de Ortopedia e Traumatologia da Universidade Federal de São Paulo.

4 - Ex-Residente do Departamento de Ortopedia e Traumatologia da Universidade Federal de São Paulo.

5 - Professor Doutor do Departamento de Ortopedia e Traumatologia da Universidade Federal de São Paulo.
} 


\section{INTRODUÇÃO}

O termo entorse é definido como uma lesão ligamentar traumática sofrida por uma articulação, devido a um movimento súbito que não chega a ocasionar luxação ${ }^{(1)}$. Estima-se que ocorra uma entorse lateral aguda do tornozelo (ELAT) a cada 10.000 pessoas por dia, sendo esta uma das lesões mais comuns do sistema musculoesquelético e também uma das mais comuns no esporte ${ }^{(2)}$.

Apesar desta elevada frequência, o diagnóstico e a conduta na ELAT representam ainda um desafio ${ }^{(3)}$ já que a avaliação da gravidade da lesão é difícil de ser estabelecida de forma definitiva no momento inicial do trauma ${ }^{(3,4)}$. Nesse cenário, o manejo da ELAT ainda é motivo de divergências verificadas em diversas publicações recentes ${ }^{(4-11)}$. Também a longo prazo, diversos estudos têm salientado um número preocupante de complicações incluindo entorses recidivantes, instabilidade do tornozelo e dor residual após a primeira entorse ${ }^{(2,3,6,12-14)}$. Isso amplifica a importância socioeconômica na ELAT, pois pode interferir aguda e cronicamente na vida profissional e social do indivíduo ${ }^{(3,6,9,10)}$.

Nos últimos cinco anos, um número considerável de estudos randomizados e controlados, bem como revisões sistemáticas, foram ou estão sendo desenvolvidos $^{(8,12,15-20)}$, tendo como foco a melhor terapêutica para as lesões de partes moles do tornozelo, incluindo a $\operatorname{ELAT}^{(6,7,9,10)}$. No entanto, vários autores afirmam que há ainda limitação metodológica dos estudos clínicos sobre a conduta na ELAT, por problemas metodológicos (número amostral, heterogeneidade dos métodos de tratamento ou por falta de padronização dos desfechos finais do tratamento) $)^{(2,9)}$. Kerkhoffs et $a l^{(18)}$, em revisão sistemática sobre imobilização funcional versus imobilização rígida e em 2006 em revisão sobre tratamento cirúrgico versus o tratamento conservador em entorse aguda do tornozelo, salientaram o número muito restrito de estudos de qualidade metodológica adequada para definir evidência de tratamento. Estudo recente comparando quatro métodos de tratamento para a entorse grave do tornozelo foi publicado ${ }^{(19)}$, mas seus resultados também foram criticados ${ }^{(20,21)}$.

Considerando-se então a frequência e a importância socioeconômica da ELAT, e as divergências da literatura, a avaliação do conhecimento e da conduta dos profissionais médicos que conduzem essa lesão é relevante tanto para se dimensionar a necessidade da discussão do tema em reuniões científicas como também para orientar ensaios clínicos futuros.
Diante do exposto conduzimos este estudo psicométrico com o objetivo primário de responder à seguinte questão: como os ortopedistas brasileiros tratam a ELAT? O objetivo secundário foi avaliar se a condução do tratamento da ELAT varia entre as categorias de profissionais estudadas.

\section{MATERIAL E MÉTODOS}

Este é um estudo descritivo. Foi elaborado um questionário (Anexo 1) com seis perguntas objetivas de múltipla escolha, que abordaram os principais aspectos relacionados ao diagnóstico, classificação, tratamento e complicações da ELAT. O questionário foi

Anexo 1 - Questionário de Avaliação sobre Entorse Lateral Aguda do Tornozelo.

\footnotetext{
1. O seu tratamento é norteado por alguma classificação?

$\square \operatorname{Sim} \square$ Não

2. Ao examinar uma entorse lateral aguda do tornozelo, você consegue classificá-la com segurança?

$\square \operatorname{Sim} \square$ Não
}

3. Em sua opinião, qual o melhor método e seu tempo médio para o tratamento da entorse lateral aguda do tornozelo com ruptura ligamentar parcial? (máximo 2 itens)

$\square$ Imobilização rígida (gessada ou órtese)

$\square$ Imobilização funcional (órteses do tipo air-cast ou de lona, esparadrapagem, bandagem ou meia elástica)

$\square$ Fisioterapia

$\square$ Medicação anti-inflamatória

Caso tenha optado pela imobilização, qual é o tempo médio necessário de utilização deste tratamento?

$\square$ até uma semana $\square$ até duas semanas $\square$ até quatro semanas

$\square$ até seis semanas $\square$ acima de seis semanas

4. Em sua opinião, qual o melhor método e seu tempo médio para o tratamento da entorse lateral aguda do tornozelo com ruptura

ligamentar total? (máximo dois itens)

$\square$ Imobilização rígida (gessada ou órtese)

$\square$ Imobilização funcional (órteses do tipo air-cast ou de lona,

esparadrapagem, bandagem ou meia elástica)

$\square$ Fisioterapia

$\square$ Cirurgia

$\square$ Medicação anti-inflamatória

Caso tenha optado pela imobilização, qual é o tempo médio necessário de utilização deste tratamento?

$\square$ até uma semana $\square$ até duas semanas $\square$ até quatro semanas

$\square$ até seis semanas $\square$ acima de seis semanas

5. Quais as complicações mais frequentes observadas em decorrência da entorse lateral aguda do tornozelo e/ou do seu tratamento? (máximo dois itens)

$\square$ Dor residual $\square$ Entorse recidivante

$\square$ Instabilidade $\square$ Lesão condral

$\square$ Trombose venosa profunda $\square$ Edema

$\square$ Incapacidade parcial ou total para as atividades prévias

6. Você é:

$\square$ Médico Residente de Programa de Ortopedia e Traumatologia

$\square$ Ortopedista Geral

$\square$ Especialista em Medicina e Cirurgia do Pé

$\square$ Especialista em Trauma Ortopédico

$\square$ Especialista em Traumatologia Desportiva

$\square$ Outro. Qual? 
avaliado e validado pela Comissão de Ensino Continuado da SBOT e disponibilizado durante o período de 15 de junho a $1^{\circ}$ de agosto de 2004, pelo Portal Oficial da Sociedade Brasileira de Ortopedia e Traumatologia (www.sbot.org.br), incluindo residentes de programa de residência em ortopedia e traumatologia e para médicos ortopedistas que atuam no Brasil. O cadastro foi programado para que somente os formulários totalmente preenchidos fossem aceitos e para que cada participante pudesse enviar apenas uma vez o questionário (dessa maneira não tivemos perdas amostrais). Para sanar qualquer dúvida ou problema dos participantes, foi disponibilizado o endereço eletrônico de dois pesquisadores. A terminologia utilizada no questionário foi definida antes que o entrevistado respondesse às perguntas.

\section{ANÁLISE ESTATÍSTICA}

A análise estatística foi realizada com a prova Q de Cochran, pelo teste de Kruskal Wallis, Mann-Whitney e Qui-quadrado para distribuição de categorias, considerando-se como valor crítico $\mathrm{p}<0,05$. Para avaliar a adequação da amostra, considerou-se uma possibilidade de concordância nas respostas de $60 \%$, um erro de estimativa máximo de $5 \%$ e um universo de 10.000 ortopedistas. Desta forma o valor mínimo amostral seria de 369 participantes.

\section{RESULTADOS}

Um total de 444 profissionais preencheram completamente o questionário e compuseram a amostra para análise, sendo 173 (39\%) ortopedistas gerais (O), 108 $(24,3 \%)$ residentes de ortopedia e traumatologia $(\mathrm{R})$, $109(24,5 \%)$ especialistas em medicina e cirurgia do pé ou em trauma ortopédico ou em traumatologia esportiva $(\mathrm{OE})$ e $54(12,2 \%)$ incluídos como ortopedistas de outras especialidades (OOE).
Os resultados sobre o conhecimento de alguma classificação e sua utilização para guiar o tratamento (Tabela 1). Verificamos que 90,8\% utilizam alguma classificação para nortear seu tratamento, mas somente $59 \%$ têm segurança ao classificar a ELAT.

Não houve diferença significativa entre as respostas dessas duas questões e a qualificação do profissional (Kruskal Wallis; $p=0,28$ e $p=0,47$, respectivamente para as questões 1 e 2).

As respostas sobre a indicação terapêutica e tempo de tratamento para a ELAT com lesão ligamentar parcial mostraram que não há consenso, visto que igual número de participantes sugeriu a imobilização funcional $(47,0 \%)$ e a imobilização rígida $(47,1 \%)$ como as intervenções mais utilizadas (Qui-quadrado, $\mathrm{p}=0,84$ ). Como a questão admitia duas opções terapêuticas, a fisioterapia foi a segunda modalidade terapêutica sugerida incluindo 30,4\% das respostas.

Quanto ao melhor método de tratamento para a ELAT com lesão ligamentar total, a imobilização rígida foi o métodomaisutilizado( $63,7 \%)$,seguidapelacirurgia(40,5\%), fisioterapia $(24,3 \%)$ e imobilização funcional $(16,2 \%)$.

Houve diferença estatisticamente significativa entre a qualificação do profissional e o tipo de indicação terapêutica para o tratamento da ELAT parcial (Kruskal Wallis, $p=0,001)$. A diferença ocorreu no grupo de ortopedista de outras especialidades (OOE) com todas as outras categorias (prova U de Mann-Witney, OOE vs. O, $\mathrm{p}=0,000 ;$ OOE $v s . \mathrm{R}, \mathrm{p}=0,001$; e OOE vs. E, $\mathrm{p}=0,000$ ), e a diferença esteve na maior frequência de indicação de imobilização funcional neste grupo de profissional em relação aos outros três grupos (Figura 1). Para o tratamento da ELAT total, não houve diferença significativa entre os profissionais (Kruskal Wallis, $\mathrm{p}=0,11$ ).

Nas figuras abaixo, são apresentadas as respostas sobre o tempo de tratamento na ELAT parcial e total (Figura 2).

Tabela 1 - Resultados das questões 1 e 2 sobre o uso de classificação para o diagnóstico da entorse lateral aguda do tornozelo e a confiança na classificação.

\begin{tabular}{c|c|c|c}
\hline Confiança na classificação & \multicolumn{3}{c}{ Tratamento baseado em classificação } \\
\hline Questão 2 & $\operatorname{Sim}$ & Questão 1 & Tão \\
\hline & $\mathrm{n}(\%)$ & $\mathrm{n}(\%)$ & $\mathrm{n}(\%)$ \\
\hline $\operatorname{Sim}$ & $254(57,2)$ & $8(1,8)$ & $262(59)$ \\
\hline Não & $149(33,6)$ & $33(7,4)$ & $182(41)$ \\
\hline Total $\mathrm{n}(\%)$ & $403(90,8)$ & $41(9,2)$ & $444(100)$ \\
\hline
\end{tabular}




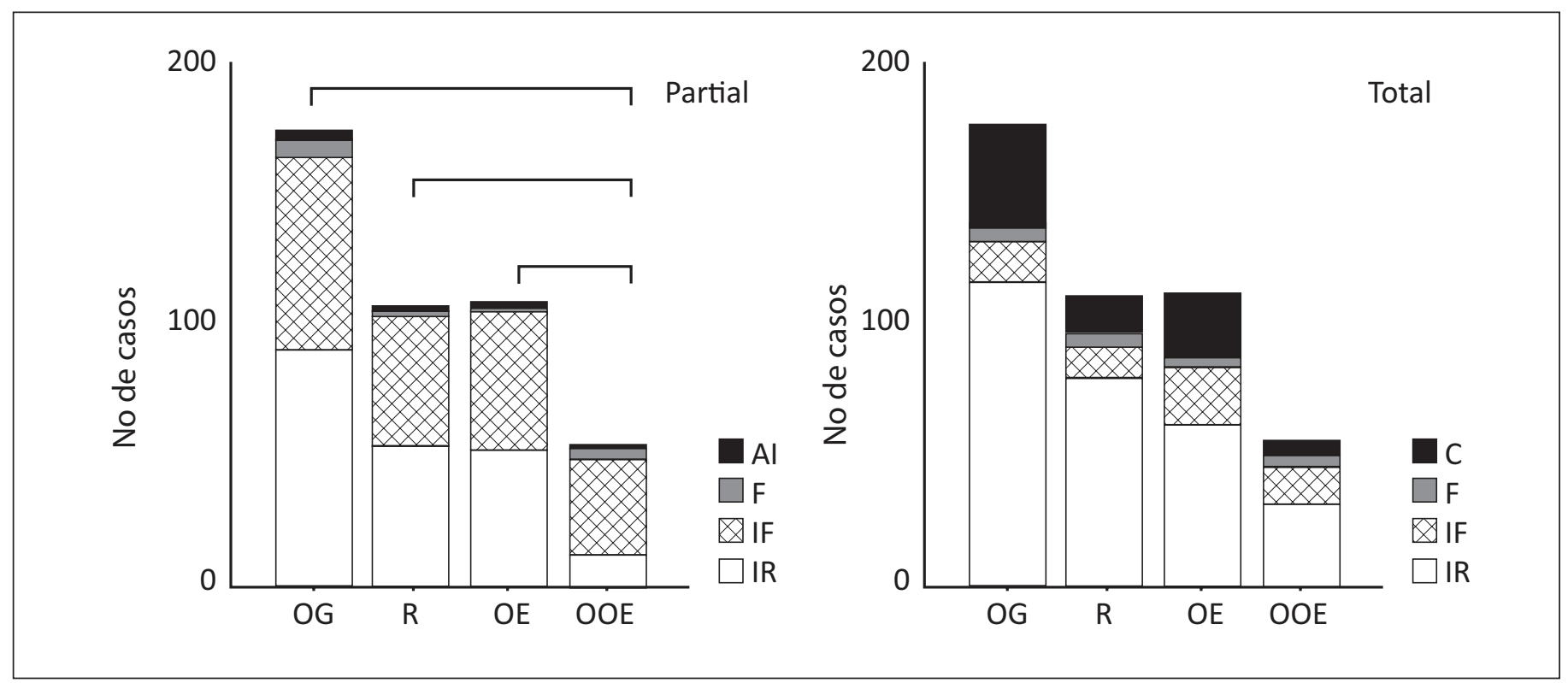

OG - Ortopedista geral; R - Residente; OE - Ortopedistas especialistas em traumatologia, traumatologia do esporte ou cirurgia do pé; OOE - Ortopedistas de outras especialidades; AI = Anti-inflamatório; C - Cirurgia; F - Fisioterapia; IF - Imobilização funcional; IR - imobilização rígida; * $-p<0,05$.

Figura 1 - Número de respostas sobre o tipo de tratamento da entorse parcial aguda do tornozelo (à esquerda) e da entorse total aguda do tornozelo (à direita) de acordo com o tipo de profissional.
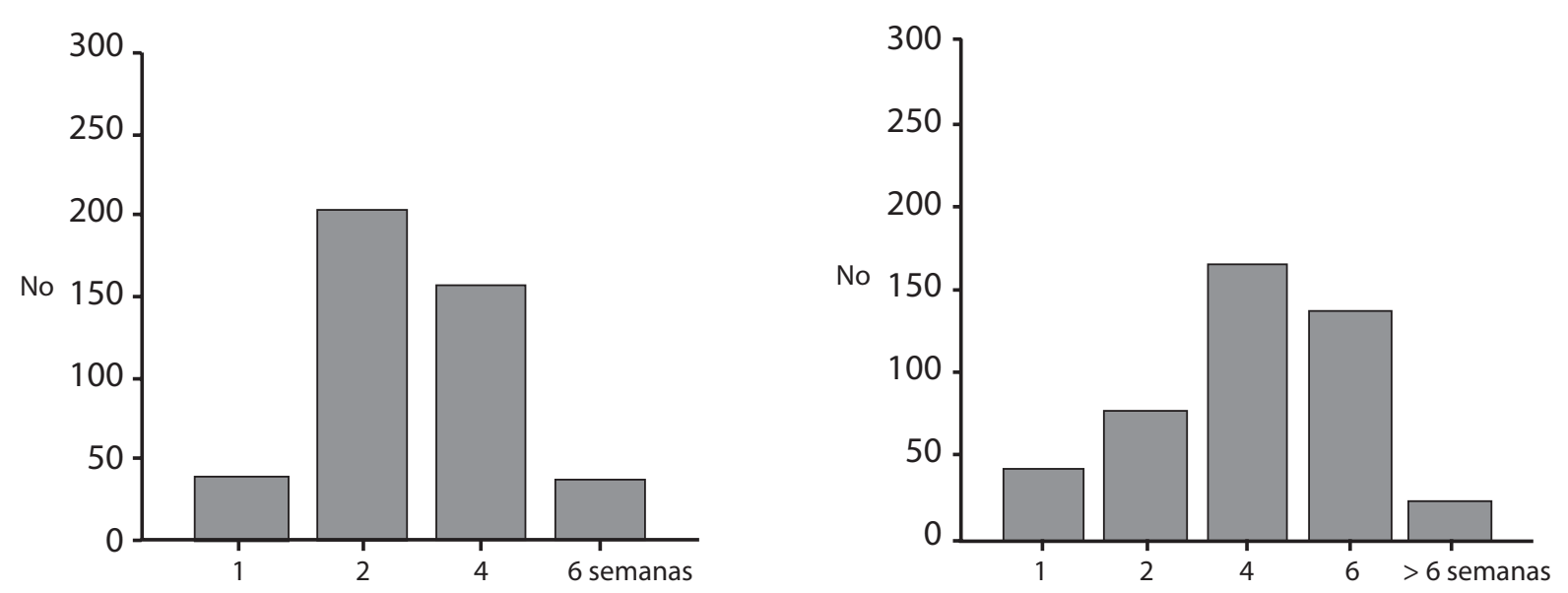

Figura 2 - Histograma do número de respostas em relação ao tempo de tratamento, em semanas, para a entorse parcial (à esquerda) e para a entorse total aguda do tornozelo (à direita).

Pode-se observar que o tempo de tratamento para a lesão parcial em até duas semanas incluiu 55,5\% das respostas e o tratamento até quatro semanas incluiu $91 \%$ das respostas, sendo a média de 2,96 $\pm 1,39$ semanas. Para a lesão total, o tempo de tratamento de até quatro semanas incluiu $64 \%$ das respostas e o tratamento de até seis semanas incluiu $95 \%$ das respostas, sendo a média de $4,1 \pm 1,79$ semanas. O tempo de tratamento foi significativamente mais longo para a ELAT total em relação à parcial (prova U de Mann Witney, $\mathrm{p}=0,00$ ), e não houve diferença significativa neste aspecto em relação à qualificação do profissional (Kruskal Wallis, $\mathrm{p}=0,23$ ).
A medicação anti-inflamatória foi significativamente mais utilizada na ruptura ligamentar parcial $(60,6 \%)$ do que na total (39\%) (prova U de Mann Witney, $\mathrm{p}=0,00)$. Não houve diferença nestas indicações entre a qualificação profissional dos entrevistados (Kruskal Wallis, $\mathrm{p}=0,71 ; \mathrm{p}=0,46)$.

Dor residual foi a complicação mais frequente $(75 \%)$ da ELAT, seguida de entorse recidivante $(43,5 \%)$ e instabilidade (34,5\%). Edema (25\%), incapacidade parcial ou total para as atividades prévias $(11,5 \%)$, lesão condral $(5,6 \%)$ e trombose venosa profunda $(0,2 \%)$ foram menos relatadas. Não houve diferença significativa desses dados em relação à qualificação do profissional (Kruskal Wallis, p =0,71). 


\section{DISCUSSÃO}

Foram encontrados na revisão de literatura dois estudos tendo como objetivo avaliar quais eram os métodos de tratamento mais utilizados nas entorses graves do tornozelo, um realizado no Reino Unido ${ }^{(22)}$ e o outro com foco na adequação à conduta fisioterápica realizado na Holanda em $2006^{(23)}$. Não foram encontrados estudos com essa finalidade na literatura nacional.

Do ponto de vista socioeconômico, devemos entender a ELAT como uma doença frequente, debilitante e onerosa. Utilizando o valor aproximado de uma ELAT para cada 10.000 pessoas/dia ${ }^{(2)}$, podemos extrapolar que na cidade de São Paulo (população estimada em cerca de 10 milhões de indivíduos, IBGE-2007) ocorram diariamente 1.000 entorses laterais do tornozelo. Como cerca da metade da população está na faixa economicamente ativa (entre 20 e 65 anos), surgiriam 500 casos novos entre trabalhadores por dia (IBGE CITIE). De acordo com os dados do presente estudo, pelo menos a metade dos pacientes seria tratada com imobilização rígida, o que impediria suas atividades laborais durante a imobilização. Supondo que eles permanecessem apenas 14 dias sem trabalhar (1.750 faltas/dia), ao final de um ano, totalizariam cerca de 1.277.500 dias não trabalhados. Considerando o valor médio do salário do trabalhador paulistano de R\$ 818,00 (Governo Federal - Programa Fome Zero), esse período de afastamento resultaria em um custo anual de aproximadamente 34 milhões de reais. Estas cifras indicam a necessidade de uma padronização de conduta, preferencialmente com base na eficácia, custo e segurança do tratamento.

A definição precisa da gravidade da ELAT é difícil de ser realizada na primeira avaliação $0^{(2,4,21,24)}$. Isto implica na decisão da terapêutica inicial, que, na maioria das vezes, é baseada em critérios não uniformes na literatura. Em 2009, Lamb et al ${ }^{(19)}$ usaram o critério "inabilidade para andar por três dias" para classificar a ELAT como grave, ao passo que, de acordo com outros autores, o critério ouro seria o encontro de anormalidades no exame físico realizado cinco dias após a entorse ${ }^{(21,24)}$. Desta forma, é ainda válida a observação de Bernett e Schirmann $^{(25)}$, que salientam que os parâmetros utilizados nas classificações disponíveis são anatômicos e de pouca aplicabilidade clínica. Essas considerações podem justificar o resultado paradoxal em nosso estudo, de que mais de $90 \%$ dos ortopedistas utilizam uma classificação no diagnóstico, porém somente $57 \%$ destes a utilizam para nortear o tratamento.
Embora os resultados sobre as opções de tratamento para a ELAT total tenham sido mais definidos $(60 \%$ na imobilização rígida e 40\% no tratamento cirúrgico), as opções para o tratamento da ELAT parcial foram claramente indefinidas ( $47 \%$ de opção tanto para a imobilização funcional quanto para a rígida). Essa variabilidade de condutas na ELAT também foi observada em estudo semelhante realizado no Reino Unido ${ }^{(22)}$. Esses resultados podem ser interpretados como adequados à falta de consenso que se observa na literatura a respeito. Kerkhoffs et al ${ }^{(18)}$, em revisão sistemática sobre imobilização funcional versus imobilização rígida em entorses agudas do tornozelo, observaram que, embora houvesse vários aspectos favoráveis à imobilização funcional, as conclusões deveriam ser interpretadas com cautela, pois não se mantiveram quando foram excluídos os estudos de pior qualidade metodológica.

Com relação à comparação do tratamento cirúrgico versus o não cirúrgico, mesmo a confecção de estudos randomizados e controlados ${ }^{(8,12,15-20)}$ ou de revisões sistemáticas $^{(6,7,9,10)}$ não levaram à conclusão sobre qual o melhor método de tratamento da ELAT. As duas mais recentes revisões de Kerkhoffs et al ${ }^{(2)}$ e a de Jones ${ }^{(9)}$ comparando o tratamento cirúrgico versus o conservador têm resultados contraditórios. No primeiro, os autores sugerem uma diferença estatisticamente favorável ao tratamento cirúrgico, enquanto a segunda conclui pela imobilização funcional. No entanto, em ambos os estudos, é salientada a falta de convicção dos resultados pela limitação metodológica dos estudos incluídos. Já o estudo de Lamb et al ${ }^{(19)}$ sugere que a imobilização rígida (gesso por 10 dias a partir do terceiro dia de lesão) foi melhor quanto à qualidade de vida e tempo de dor, embora os resultados tenham sido criticados tanto pela falta de um maior controle sob as outras formas de tratamento $^{(21)}$ como pelo critério de seleção dos casos que não considerou o exame tardio do tornozelo como método ideal para a seleção dos $\operatorname{casos}^{(24)}$.

No tratamento das ELAT parciais, a segunda modalidade mais escolhida foram os anti-inflamatórios não hormonais (AINH). Em revisão recente, há indícios de que essas medicações possam melhorar a função articular e acelerar a cura da lesão, porém não apresentam diminuição da dor e do edema ${ }^{(11)}$.

Os resultados acerca do tempo de tratamento foram homogêneos, com a maioria variando entre quatro e seis semanas, sendo significantemente maior nos casos de lesão total dos ligamentos. Esses dados corroboram a prática comum diária dos pronto-socorros, mas apesar 
desse "consenso tácito", não foram encontrados dados consistentes na literatura sobre esse assunto ${ }^{(26)}$.

$\mathrm{Na}$ avaliação das complicações mais frequentes, a maioria dos ortopedistas indicou a dor residual $(75 \%)$ e, em seguida, as entorses recidivantes $(43,5 \%)$ e a instabilidade $(34,5 \%)$. Alguns estudos recentes avaliaram a evolução a curto e longo prazos da ELAT (anos após) e mostraram que, após um ano ou até três anos após o trauma, há elevada prevalência de dor (33\% a $43 \%)$, recorrência da entorse (3\% a 34\%) e impossibilidade de andar por distância maior que uma milha $(1,6 \mathrm{~km})^{(6,13,14)}$. Dessa forma, os resultados do presente estudo são congruentes quanto aos tipos de complicações encontradas na evolução da ELAT.

Alguns aspectos merecem ser relevados diante da análise dos nossos dados. Por se tratar de estudo envolvendo questionário que demanda evocação da memória, esses podem ser super/subestimados, além de terem sido obtidos via internet, o que é fonte de viés de seleção. Fato que conta como fator diminuidor deste viés é o significante número amostral. É importante salientar que estes achados refletem a opinião dos ortopedistas e não significam que sejam um guia para o tratamento.

\section{CONCLUSÕES}

Os dados desse estudo mostraram que os ortopedistas brasileiros encontram dificuldade em classificar a ELAT (apenas 57\% o fazem com segurança). Divergem quanto à melhor opção terapêutica, nas parciais $(47 \%$ utilizam imobilização funcional e $47,1 \%$ rígida); porém, concordam em relação às lesões totais $(63,7 \%$ indicam imobilização funcional e 40,5\% cirurgia). Os ortopedistas brasileiros consideram a dor como a principal complicação da ELAT (75\%) e concordam sobre o tempo de imobilização da ELAT parcial e total.

\section{Implicações para futuras pesquisas}

Há necessidade de estudos de qualidade para desenvolvimento de um guia para o manejo uniforme destas lesões. O desenvolvimento de uma classificação objetiva, reprodutível e com aplicação clínica na entorse lateral aguda do tornozelo, deve ser foco de pesquisas futuras.

\section{REFERÊNCIAS}

1. Kannus P, Renström P. Treatment for acute tears of the lateral ligaments of the ankle. Operation, cast, or early controlled mobilization. J Bone Joint Surg Am. 1991;73(2):305-12.

2. Kerkhoffs GM, Rowe BH, Assendelft WJ, Kelly K, Struijs PA, van Dijk CN. Immobilisation and functional treatment for acute lateral ankle ligament injuries in adults. Cochrane Database Syst Rev. 2002;(3):CD003762.

3. Abbassian A, Thomas R. Ankle ligament injuries. Br J Hosp Med (Lond) 2008;69(6):339-43.

4. Van Dijk CN, Lim LS, Bossuyt PM, Marti RK. Physical examination is su fficient for the diagnosis of sprained ankles. J Bone Joint Surg Br. 1996;78 (6):958-62

5. McKeon PO, Mattacola CG. Interventions for the prevention of first time and recurrent ankle sprains. Clin Sports Med. 2008;27(3):371-82.

6. Margo KL. Review: many adults still have pain and subjective instability at 1 year after acute lateral ankle sprain. Evid Based Med. 2008;13(6):187.

7. Bleakley CM, McDonough SM, MacAuley DC. Some conservative strategies are effective when added to controlled mobilisation with external support after acute ankle sprain: a systematic review. Aust J Physiother. 2008;54(1):7-20

8. Mohammadi F. Comparison of 3 preventive methods to reduce the recurrence of ankle inversion sprains in male soccer players. Am J Sports Med. 2007;35(6):922-6

9. Jones $\mathrm{MH}$, Amendola AS. Acute treatment of inversion ankle sprains: immobilization versus functional treatment. Clin Orthop Relat Res. 2007;455:169-72.

10. Fong DT, Hong Y, Chan LK, Yung PS, Chan KM. A systematic review on ankle injury and ankle sprain in sports. Sports Med. 2007;37(1):73-94

11. Ivins D. Acute ankle sprain: an update. Am Fam Physician. 2006;74(10): 1714-20.

12. Hupperets MD, Verhagen EA, van Mechelen W. The 2BFit study: is an unsupervised proprioceptive balance board training programme, given in addition to usual care, effective in preventing ankle sprain recurrences? Design of a randomized controlled trial. BMC Musculoskelet Disord. 2008;9:71.

13. Haywood KL, Hargreaves J, Lamb SE. Multi-item outcome measures for lateral ligament injury of the ankle: a structured review. J Eval Clin Pract. 2004;10(2):339-52.

14. Braun BL. Effects of ankle sprain in a general clinic population 6 to 18 months after medical evaluation. Arch Fam Med. 1999;8(2):143-8.
15. Bleakley CM, O'Connor S, Tully MA, Rocke LG, Macauley DC, McDonough SM. The PRICE study (Protection Rest Ice Compression Elevation): design of a randomized controlled trial comparing standard versus cryokinetic ice applications in the management of acute ankle sprain [ISRCTN13903946]. BMC Musculoskelet Disord. 2007;8:125

16. Van Rijn RM, van Os AG, Kleinrensink GJ, Bernsen RM, Verhaar JA, Koes BW, et al. Supervised exercises for adults with acute lateral ankle sprain: a randomised controlled trial. Br J Gen Pract. 2007;57(543):793-800.

17. Petrella RJ, Petrella MJ, Cogliano A. Periarticular hyaluronic acid in acute ankle sprain. Clin J Sport Med. 2007;17(4):251-7.

18. Kerkhoffs GM, Handoll $\mathrm{HH}$, de Bie R, Rowe $\mathrm{BH}$, Struijs PA. Surgical versus conservative treatment for acute injuries of the lateral ligament complex of the ankle in adults. Cochrane Database Syst Rev. 2002;(3):CD000380.

19. Lamb SE, Marsh JL, Hutton JL, Nakash R, Cooke MW; Collaborative Ankle Support Trial (CAST Group). Mechanical supports for acute, severe ankle sprain: a pragmatic, multicentre, randomised controlled trial. Lancet. 2009;373(9663):575-81.

20. Cooke MW, Marsh JL, Clark M, Nakash R, Jarvis RM, et al. Treatment of severe ankle sprain: a pragmatic randomised controlled trial comparing the clinical effectiveness and cost-effectiveness of three types of mechanical ankle support with tubular bandage. The CAST trial. Health Technol Assess. 2009;13(13): 1-121.

21. Kerkhoffs GM, van den Bekerom MP, Struijs PA, van Dijk CN. 10-day below-knee cast for management of severe ankle sprains. Lancet. 2009;373(9675):1601.

22. Cooke MW, Lamb SE, Marsh J, Dale J. A survey of current consultant practice of treatment of severe ankle sprains in emergency departments in the United Kingdom. Emerg Med J. 2003;20(6):505-7.

23. Leemrijse CJ, Plas GM, Hofhuis $\mathrm{H}$, van den Ende $\mathrm{CH}$. Compliance with the guidelines for acute ankle sprain for physiotherapists is moderate in the Netherlands: an observational study. Aust J Physiother. 2006;52(4):293-9.

24. Van Rijn RM, van Middelkoop M. 10-day below-knee cast for management of severe ankle sprains. Lancet. 2009 May 9;373(9675):1601

25. Bernett $P$, Schirmann A. [Acute sporting injuries of the ankle joint (author's transl)]. Unfallheilkunde. 1979;82(4):155-60.

26. Yamamoto $\mathrm{H}$, Ishibashi $\mathrm{T}$, Muneta $\mathrm{T}$, Furuya $\mathrm{K}$. Nonsurgical treatment of lateral ligament injury of the ankle joint. Foot Ankle. 1993;14(9):500-4 